\title{
Eventos Cerebrovasculares: manejo y prevención secundaria
}

Treatment and secondary prevention of stroke: evidence, costs, and effects on individual populations Graeme J Hankey, Charles P Warlow. Lancet 1999; 354: 1457-1463

\section{Introducción}

El manejo tanto preventivo como terapéutico de la enfermedad cerebrovascular muestra una gran variabilidad cuando se comparan diferentes centros asistenciales o distintas regiones. Existen varias razones que pueden explicar esta situación. Ya hemos comentado anteriormente en Evidencia ${ }^{1}$ la dificultad en la generalización automática de los resultados de los estudios aleatorizados cuando la calidad de la intervención analizada es diferente a la de nuestro medio (por ej: muy diferente mortalidad perioperatoria de la endarterectomía carotídea en los ensayos clínicos que en condiciones habituales). En otras ocasiones los resultados finales considerados son confusos o de relativa trascendencia clínica (como la reducción de Accidentes Isquémicos Transitorios en vez de accidentes cerebrovasculares o muerte). Finalmente, muchos estudios tuvieron limitaciones como un insuficiente o bajo poder estadístico. En gran medida, estos inconvenientes se superan mediante las revisiones sitemáticas de la literatura como las de la Colaboración Cochrane 2 o la US Agency for Healthcare Research and Quality (antes Agency for Health Care Policy and Research) ${ }^{3}$. El objetivo del presente trabajo fue revisar la mejor evidencia para la prevención y el manejo del ACV, y analizar comparativamente la efectividad y costo de las diversas intervenciones. Cabe aclarar que los costos reportados en el presente informe corresponden a los vigentes en países desarrollados, pudiendo existir diferencias significativas en otros medios.

\section{Métodos}

Búsqueda de revisiones sistemáticas y ensayos clínicos controlados randomizados sobre tratamiento y prevención secundaria del ACV en Cochrane Library y US National Library of Medicine's Medline

\section{INTERVENCIONES EN EL ACCIDENTE CEREBROVASCULAR (ACV) "Unidades de Stroke"}

Las unidades de ACV (Stroke en inglés) surgen con una idea similar a la de la unidad coronaria pero para el manejo intensivo de los pacientes con ACV. Estas unidades específicas redujeron la mortalidad y mejoraron la funcionalidad al año del ACV con respecto a los cuidados médicos generales convencionales. Con tratamientos generales la proporción combinada de mortalidad y dependencia (limitación ostensible de la autonomía en las funciones de la vida diaria) fue del $62 \%$ vs. un $56,4 \%$ en las unidades de ACV. Esto implica un RRR (reducción del riesgo relativo) del $9 \%$, una RRA (reducción del riesgo absoluto) del 5,6\% y un NNT (número de pacientes necesario para tratar) de 18. No tenemos datos disponibles para un adecuado análisis de costos de las unidades de ACV. Como en estas unidades los días promedio de internación son similares a los de una internación general, los determinantes del costo específico de las mismas los constituyen la infraestructura y el personal requeridos.

\section{Aspirina (AAS)}

La aspirina en dosis diaria de 160 a $300 \mathrm{mg}$ disminuye la mortalidad y la dependencia de un $47 \%$ a un $45,8 \%$. Es mal tolerada o está contraindicada en aproximadamente un $5 \%$ de la población. El NNT para mortalidad o dependencia es de 83 pacientes (tratando a 83 pacientes con un evento vasculoencefálico agudo con AAS evito una muerte o invalidez con vida dependiente). El costo de tratamiento en agudo para prevenir una muerte o dependencia al año (300 mg por día por 2 semanas a partir del ACV a 83 pacientes) es de aproximadamente $\$ 60$.

\section{Trombolisis}

Este es un tópico más controvertido. La trombolisis intravenosa dentro de las 6 hs. de la instalación del cuadro agudo reduciría la mortalidad y dependencia del $62,7 \%$ al $56,4 \%$. Sin embargo, solo el $10 \%$ de los pacientes podrían realizarla (rápido acceso a centro asistencial, tomografía disponible para descartar sangrado encefálico, sin contraindicaciones para terapia trombolítica). El costo aproximado de tratamiento con alteplase para prevenir una muerte o vida dependiente es de aproximadamente $\$ 36.000$.

\section{Heparina}

Una reciente revisión sistemática ${ }^{4}$ no halló disminución de la mortalidad o dependencia del ACV agudo tratado rutinariamente con heparina: RRR 1\%; (IC 95\%: $-5 \%$ a $6 \%$ ). Por lo tanto su uso rutinario no es aconsejado.

\section{PREVENCION SECUNDARIA}

\section{Control de la Presión Arterial}

Existe abundante evidencia a favor del control de la hipertensión arterial (HTA) como intervención eficaz para la disminución de la morbimortalidad por ACV. La reducción de la tensión arterial diastólica en 5$6 \mathrm{~mm} \mathrm{Hg}$ y la sistólica en 10-12 mm Hg por 2-3 años en pacientes hipertensos con enfermedad vasculocerebral (TIA/ $\mathrm{ACV}$ ), reduciría el riesgo anual de ACV de un 7 a un 4,8\%. Esto implica un NNT de 18 . El costo anual es muy variable de acuerdo a las drogas prescriptas (entre $\$ 1350$ y $\$ 18.000$ para prevenir $1 \mathrm{ACV})$.

\section{Tabaquismo}

Estudios observacionales sugieren que el tabaquismo aumenta de 2 a 5 veces el riesgo de AIT y ACV. El NNT de abandonar el hábito de fumar es 43. Si los fumadores víctimas de un evento vasculocerebral requirieran 3 meses de parches de nicotina para abandonar el hábito, la prevención de un episodio costaría aproximadamente $\$ 20.000$.

\section{Reducción del Colesterol plasmático}

Existe evidencia indirecta (estudios en pacientes coronarios) que la terapia hipocolesterolemizante con estatinas reduce el riesgo de ACV en aproximadamente un $24 \%$. ${ }^{5-6}$ Si se utilizara simvastatina $20 \mathrm{mg}$ /día o pravastatina $20-40 \mathrm{mg} /$ día, el costo por paciente por año sería de aproximadamente $\$ 500$ y serían necesarios $\$ 29.000$ al año para prevenir $1 \mathrm{ACV}$.

\section{Antiagregantes}

La aspirina en dosis de $75 \mathrm{mg} /$ día o mayores disminuye el riesgo relativo de nuevos eventos en un $13 \%$ en administrado a pacientes con antecedentes de AIT o ACV. El NNT de la aspirina es de 100. El clopidogrel es algo más eficaz que la aspirina, ${ }^{7}$ con un NNT de 62. La adición de dipiridamol a la aspirina es controversial, si bien un meta-análisis sugiere mayor eficacia 8 con un NNT de 53. El costo anual de la aspirina para prevenir un evento vasculocerebral es de aproximadamente $\$ 2.000$; $\$ 74.400$ el del clopidogrel y $\$ 17.500$ para AAS+dipiridamol.

\section{Anticoagulantes}

La anticoagulación oral con un RIN (razón internacional normatizada) de 3 en pacientes fibrilados disminuye el riesgo anual de ACV de un 12 a un $4 \%$. El 0,5 a $0,8 \%$ de los pacientes sufren hemorragias mayores. Aproximadamente un $20 \%$ de los pacientes con TIA/ ACV tienen indicación de anticoagulación. El NNT para evitar un ACV es de 13 y el costo por cada ACV evitado es de aproximadamente $\$ 850$.

\section{Endarterectomia Carotídea (EC)}

La EC en pacientes con AIT reciente en territorio carotídeo o ACV menor, o en los portadores de estenosis carotídea severa y buenos candidatos quirúrgicos, disminuye el riesgo de ACV a los 3 años de 26,5 a $14,9 \%$. 9 Solo el $8 \%$ de los pacientes encuadran en los criterios de in- 
clusión previos. El NNT para evitar un evento es 27 . El costo anual aproximado para evitar un ACV por los siguientes 3 años es de $\$ 130.000$.

\section{CONCLUSIONES}

De la evidencia disponible surgen las siguientes intervenciones avaladas en el manejo del ACV:

- Desarrollo de Unidades de ACV y equipos terapéuticos multidisciplinarios

- AAS $300 \mathrm{mg} /$ día

- Trombolisis en pacientes con indicaciones precisas y medios propicios

\section{Prevención Secundaria:}

- AAS a menor dosis (75 mg/día)

- Abandono del tabaco

- Tratamiento óptimo de la HTA (diuréticos en HTA no complicada)

- Anticoagulación en caso de fibrilación auricular

- Estatinas en pacientes hipercolesterolémicos y/o coronarios sintomáticos

- Individualización de pacientes de alto riesgo que podrían beneficiarse de intervenciones más costosas y de indicaciónes más precisas como sustitución de AAS con clopidogrel, adición de dipiridamol, y endarterectomía cartotídea.

\section{Comentario}

La presente revisión constituye un importante aporte sintetizador de la evidencia disponible sobre las diferentes intervenciones factibles ante un paciente con enfermedad vasculocerebral aguda. Encontramos que la evidencia en favor de las intervenciones más cercanas a la prevención son las más sedimentadas, eficaces, efectivàs e indiscutidas. Las intervenciones terapéuticas en cambio, son en general más costosas y la evidencia que las sustenta es más heterogénea en cuanto al rendimiento hallado. Así, ya no caben dudas de la necesidad de controlar los factores de riesgo vascular tanto para la prevención primaria como secundaria del ACV, así como está ampliamente suscripta la antiagregación plaquetaria. En este sentido episódicamente aparecen ciertas controversias en las dosis o tipo de drogas a utilizar (aspirina a altas o bajas dosis; ticlopidina o clopidogrel; adición ${ }^{7}$ o no de dipiridamol (Tolouse Study, French AICLA, Canadian-American Study). No obstante sabemos que con una u otra técnica, debemos antiagregar a los pacientes vasculoencefálicos. No ocurre lo mismo con intervenciones terapéuticas tales como la fibrinolisis que apareció como contraproducente en ciertos estudios ${ }^{10}$ y como beneficiosa en otros ${ }^{11}$, o la endarterectomía carotídea, de probada eficacia en un subgrupo de pacientes, pero no universalizable en sus indicaciones y discutida en pacientes asintomáticos. Es importante recalcar que los costos referidos en la presente guía son estimativos y su fuente es la del artículo analizado por lo que podrían diferir en algunos casos sustancialmente en nuestro medio. No obstante son útiles al menos como patrón de comparación relativa entre las diferentes intervenciones preventivo-terapéuticas. Otra consideración remarcable que surge del análisis de cada intervención es que el impacto global de la misma en la salud de la población analizada radica en gran medida en cúan generalizable es. Por ejemplo, una intervención potencialmente muy eficaz como la trombolisis, al ser pasible de indicación en una minoría de los pacientes con ACV, redunda en un impacto global sustancialmente menor que otra intervención individualmente más debil, pero casi universal como lo es la antiagregación. Algo similar ocurre con las unidades de ACV, que constituyen una intervención indicada universalmente, pero disponible solo en selectos centros por lo que los pacientes pasibles de beneficiarse con la misma son una ínfima minoría. Finalmente para mejorar la costoefectividad de las intervenciones costosas como el clopidogrel o la endarterectomía carotídea, deben extremarse los esfuerzos por detectar la subpoblación de pacientes que más se beneficiará de estas intervenciones, con lo que disminuirá tanto el costo anual del tratamiento como el NNT.

\section{Dr. Alejandro Regueiro}

Sistema de Protección Médica

\section{Referencias}

1. Regueiro A. Editorial: Del ensayo clínico al consultorio. Evidencia en Atención Primaria. 1999 Marzo-Abril Vol.2 Nro.2: 34-35.

2. http://hiru.mcmaster.ca/COCHRANE/DEFAULT.HTM

3. http://www.ahrq.gov/

4. Sandercock PAG, Gubitz G, Counsell C, et al. Immediate anticoagulant therapy for acute ischaemic stroke I: a systematic review of 21 randomized trials of anticoagulant vs. Control, including 23.374 patients. Stroke 1999; 30: 248 (abstr).

5. Bucher HC, Griffith LE, Guyatt GH. Effect of HMG Co A reductase inhibitors on stroke: a meta-analysis of randomised, controlled trials. Ann Intern Med 1998; 128 : 89- 95.

6. Plehn JF, Davis BR, Sacks FM, et al, for the CARE investigators. Reduction of stroke incidence after myocardial infarction with pravastatin. The Cholesterol and Recurrent Events (CARE) Study. Circulation 1999; 99: 216-223.

7. Hankey GJ, Sudlow CLM, Dunbabin DW. Thienopyridine derivatives (ticlopidine, clopidogrel) vs. Aspirin in the secondary preevntion of stroke and other impor tant vascular events among high risk patientes (protocol for a Cochrane Review) in: The Cochrane Library. Oxford: Update Software 1999. Updated quarterly.

8. Diener HC, Cunha L, Forbes C, et al. European Stroke Prevention Study 2: dipyridamole and acetylsalicylic acid in the secondary prevention of stroke. J Neurol Sci 1996; 143: 1-13.

9. European Carotid Surgery Trialists' Collaborative Group. Randomised trial of endarterectomy for recently symptomatic carotid stenosis: final results of the MRC European Carotid Surgery Trial (ECST). Lancet 1998; 351: 1379-1387.

10. Multicenter Acute Stroke Trial Italy Study (MAST-I) Group. Steptokynasa for the treatment of acute ischaemic stroke. Lancet 1995; $346: 1509-1514$.

11. Wardlaw JM, Yamaguchi T, del Zoppo G. Thrombolytic therapy vs. control in acute ischaemic stroke (Cochrane review). In: The Cochrane Library, Oxford: Update Software. Updated quarterly. 